\title{
Solute channels of the outer membrane: from bacteria to chloroplasts
}

\author{
Daniela Duy, Jürgen Soll and Katrin Philippar* \\ Department Biologie 1, Botanik, Biochemie und \\ Physiologie der Pflanzen, Ludwig-Maximilians- \\ Universität München, Menzingerstr. 67, D-80638 \\ München, Germany \\ *Corresponding author \\ e-mail: philippar@|rz.uni-muenchen.de
}

\begin{abstract}
Chloroplasts, unique organelles of plants, originated from endosymbiosis of an ancestor of today's cyanobacteria with a mitochondria-containing host cell. It is assumed that the outer envelope membrane, which delimits the chloroplast from the surrounding cytosol, was thus inherited from its Gram-negative bacterial ancestor. This plastid-specific membrane is thus equipped with elements of prokaryotic and eukaryotic origin. In particular, the membrane-intrinsic outer envelope proteins (OEPs) form solute channels with properties reminiscent of porins and channels in the bacterial outer membrane. OEP channels are characterised by distinct specificities for metabolites and a quite peculiar expression pattern in specialised plant organs and plastids, thus disproving the assumption that the outer envelope is a non-specific molecular sieve. The same is true for the outer membrane of Gramnegative bacteria, which functions as a permeability barrier in addition to the cytoplasmic membrane, and embeds different classes of channel pores. The channels of these prokaryotic prototype proteins, ranging from unspecific porins to specific channels to ligand-gated receptors, are exclusively built of $\beta$-barrels. Although most of the OEP channels are formed by $\beta$-strands as well, phylogeny based on sequence homology alone is not feasible. Thus, the comparison of structural and functional properties of chloroplast outer envelope and bacterial outer membrane channels is required to pinpoint the ancestral OEP 'portrait gallery'.
\end{abstract}

Keywords: endosymbiosis; metabolite channel; outer envelope protein; plant; plastid; porin.

\section{Introduction: plastids and their envelope membranes}

Chloroplasts, which originated from ancient free-living cyanobacteria (Moreira et al., 2000; Palmer, 2000), are the site of photosynthesis and thus represent the basis for all life dependent on atmospheric oxygen and carbohydrate supply. In the cells of plants and green algae, chloroplasts, which are enclosed by outer and inner envelope membranes, therefore represent unique and essential organelles. Both envelope membranes are distinguishable by their structure, function and biochemical properties, but also co-operate in the synthesis of lipids and in protein translocation (Douce and Joyard, 1990; Joyard et al., 1998). In higher plants, however, chloroplasts represent only one type of the plastid organelle family. Proplastids in meristem tissue and etioplasts in dark-grown plantlets develop during biogenesis into the mature, autotrophic chloroplast of the green leaf. In contrast, storage plastids are heterotrophic organelles that convert photosynthates derived from source tissue into storage compounds that can be mobilised during plant development (see Bowsher and Tobin, 2001; Lopez-Juez and Pyke, 2005 for an overview). Amyloplasts (starch) in the endosperm of seeds and in cotyledons, tubers or fruits, elaioplasts (oil) in seeds of oilseed plants, and chromoplasts (carotenoids) in flowers and fruits represent the major storage-type plastids. Root plastids, with major functions in the oxidative pentose phosphate pathway and nitrogen assimilation, are also members of the socalled non-green plastids (for a review on plastid differentiation, see Waters and Pyke, 2005).

Thus, the plastid organelle family conducts vital biosynthetic functions in every plant cell (for an overview see Weber et al., 2005; Tetlow et al., 2005). First and foremost, chloroplasts carry out photosynthesis, resulting in the release of $\mathrm{O}_{2}$ and the conversion of atmospheric $\mathrm{CO}_{2}$ into carbohydrates that are directly used for starch biosynthesis in the chloroplast stroma, as well as sucrose synthesis in the cytosol. Furthermore, chloroplasts are involved in the biosynthesis of many metabolites and compounds such as isoprenoids (e.g., carotenoids, and sterols), tetrapyrroles (e.g., haem and chlorophyll), nucleic acids, aromatic and non-aromatic amino acids, polyphenols, and lignins. Nitrite produced in the cytosol during nitrogen assimilation is transported into the plastid and reduced to ammonia by plastidic nitrite reductase. All subsequent reactions of primary assimilation and amino acid synthesis take place within plastids of photosynthetic and non-photosynthetic tissue. In plants, de novo synthesis of fatty acids is carried out in plastids, which are also the site for sulfate reduction and biosynthesis of iron-sulfur clusters.

These manifold biosynthetic functions of plastids require different selective transport mechanisms across the envelope membranes for the exchange of carbohydrates, organic nitrogen and sulfur compounds. Furthermore, plastids take up inorganic cations $\left(\mathrm{K}^{+}, \mathrm{Na}^{+}, \mathrm{Mg}^{2+}\right.$, $\left.\mathrm{Ca}^{2+}, \mathrm{Fe}^{2+}, \mathrm{Cu}^{2+}, \mathrm{Mn}^{2+}, \mathrm{Zn}^{2+}\right)$, anions $\left(\mathrm{NO}_{2}^{-}, \mathrm{SO}_{4}{ }^{2-}, \mathrm{PO}_{4}{ }^{3-}\right)$, and a variety of organic biosynthetic pathway interme- 
diates, such as acetate and dicarboxylic acids, to fulfil their biosynthetic tasks.

In this review we describe transport pathways for solutes and metabolites across the outer envelope of plastids and the outer membrane of Gram-negative bacteria. Since these prokaryotes are the evolutionary progenitors of plastids, their set of outer membrane-integral channels and porins might serve as a blueprint for the situation in the plastidic outer envelope. For a detailed overview of transporters in the inner envelope of chloroplasts, as well as other plant organelles, we refer to the recent reviews of Weber (2004), Weber et al. (2005), and Philippar and Soll (2007).

\section{Outer membrane channels and porins in plant plastids and mitochondria: evolutionary aspects}

Gram-negative bacteria represent the evolutionary progenitors of plastids and mitochondria. As described below, they contain several different classes of porins and channels in their outer membrane. Chloroplasts (from ancient cyanobacteria) and mitochondria (from proteobacterial ancestors), however, most likely originated from two independent endosymbiotic events. For instance, this was demonstrated by analysis of the phylogenetic origin of the alternative oxidase in mitochondria and chloroplasts, as well as of the outer membrane localised $\beta$-barrel transporters of the Omp85 family (Atteia et al., 2004; Bredemeier et al., 2007). Thus, it seems possible that the outer membranes of chloroplasts and mitochondria are equipped with different channel and porin types, depending on the metabolic needs of the organelle. Whereas in mitochondria with the voltage-dependent anion channel (VDAC, see below) only one non-specific porin, which to some extent can be regulated by metabolites, substrates and nucleotides, was retained, the situation in plastids is more complex, reflecting their integration in the biosynthetic pathways of many metabolites essential for the plant cell. Thus, as described below, structural and functional elements or regulation of gene expression of several of the nucleusencoded outer envelope protein (OEP) channels in the plastid outer envelope are reminiscent of the outer membrane channels and porins in Gram-negative bacteria. However, the plastid outer envelope is not only equipped with elements of prokaryotic origin, but also contains proteins derived from the eukaryotic host.

During evolution, most of the ancient cyanobacterial genome information was transferred to the nucleus (Martin et al., 2002; Leister, 2003, 2005). However, chloroplasts, which retained a prokaryote-type genetic system, amongst others, most likely inherited phytochromes (Pepper, 1998) and carotenoids from cyanobacteria (Hoiczyk and Hansel, 2000). Furthermore, with synToc75 from Synechocystis (Bölter et al., 1998) and cyanobacterial orthologues of Tic20, Tic22, and Tic55 (Reumann et al., 2005), at least part of the protein import machinery of chloroplasts, Toc (translocon at the outer envelope) and Tic (translocon at the inner envelope of chloroplasts), was recruited from the cyanobacterial ancestor. For pro- tein import into chloroplasts, see Soll and Schleiff (2004) and Vothknecht and Soll (2005) and references therein. However, whereas Toc75 builds the channel for protein translocation into chloroplasts, its progenitor, Omp85, functions as an export pore and/or during insertion of outer membrane proteins (OMPs) in bacteria, as well as in mitochondria (Bos and Tommassen, 2004; Gentle et al., 2004, 2005; Bredemeier et al., 2007). For a more detailed overview of plastid biogenesis, refer to Moreira et al. (2000), Palmer (2000), Raven and Allen (2003), Vothknecht and Soll (2005), and Lopez-Juez (2007).

\section{VDAC, the porin in the outer membrane of mitochondria}

The outer membrane of mitochondria is freely permeable to solutes up to a size of $4-5 \mathrm{kDa}$ (Benz, 1994), which is due to the presence of a porin similar to the "classical porins' in the outer membrane of Gram-negative bacteria (see below). In mitochondria, this $\beta$-barrel pore, with a molecular weight of approximately $30-36 \mathrm{kDa}$, is called VDAC. Although primary amino-acid sequences may vary, secondary structural elements, including an $\mathrm{N}$-terminal $\alpha$-helical domain and $16 \beta$-sheets, are highly conserved among eukaryotic VDAC porins. While the channel pore with an apparently large diameter (up to $3 \mathrm{~nm}$; see Nikaido, 2003 and references therein) is built by the $\beta$-sheets, the $\alpha$-helical domain is involved in voltage sensing and gating (Mannella, 1998). In lipid bilayer membranes, VDAC forms an aqueous channel with low substrate selectivity and high single-channel conductance (approx. $4 \mathrm{nS}$ in $1 \mathrm{M} \mathrm{KCl}$ ) and thus functions more or less as a size-exclusion filter. However, evidence has accumulated that gating of VDAC is regulated by several factors such as NADH or proteins localised in the intermembrane space, indicating a complex mechanism of solute exchange in vivo (Colombini et al., 1987; Holden and Colombini, 1993; Zizi et al., 1994; Rostovtseva and Colombini, 1997; Van der Heiden et al., 2000). The first plant VDAC was identified electrophysiologically in purified outer membranes of pea mitochondria (Schmid et al., 1992). Since then, mitochondrial porins have been isolated and characterised from several plant species (Aljamal et al., 1993; Blumenthal et al., 1993; Abrecht et al., 2000a,b; Godbole et al., 2003; Wandrey et al., 2004).

\section{A porin in the outer envelope of plastids?}

The permeability of isolated chloroplasts was first examined by electron microscopy and distribution of radioactively labelled compounds (Heldt and Sauer, 1971). These early studies were followed by electrophysiological approaches after reconstitution of outer envelope membranes into artificial lipid bilayers (Flügge and Benz, 1984) and by applying the patch-clamp technique on isolated intact organelles (Pottosin, 1992, 1993). In summary, the results obtained suggested that the plastidic outer envelope is equipped with a general diffusion pore related to the porin VDAC from mitochondria. Therefore, it has long been assumed that the outer envelope of plastids is freely permeable for low-molecular-weight solutes of up to $10 \mathrm{kDa}$. Correspondingly, it was believed that the osmotic barrier against the cytosol is formed exclusively by the 
inner envelope. Subsequently, Fischer et al. (1994) localised the mitochondrial VDAC to non-green plastids of pea roots, but not to chloroplasts of green leaves. However, recent studies on subcellular distribution of the VDAC protein between plastids and mitochondria in green and non-green tissues showed that VDAC is present in mitochondria only (Clausen et al., 2004). Thus, since this general diffusion porin in plastids could not be identified on the molecular level, the identity of a VDAClike porin in the outer envelope of chloroplasts is equivocal.

\section{Solute channels in the outer envelope of plastids}

In contrast, transport capacity and selectivity could be assigned to several OEPs. Thus, the outer envelope of chloroplasts is equipped with low-affinity, highly specific channels (OEP16), the substrate- and ATP-gated OEP21, and the specifically expressed, but rather unspecific porin-like OEP24, reflecting the entire spectrum of their bacterial ancestors (for details see below). However, while the inner envelope transporters show distinct substrate specificity, it remains a matter of debate to what extent transport through channels of the outer envelope is regulated in vivo (for a discussion see Soll et al., 2000; Flügge, 2000; Bölter and Soll, 2001). In general, recent research in the field suggests that quite a high number of as yet uncharacterised membrane transporters exist in all plant organelles (Philippar and Soll, 2007). Thus, the view of a non-specific diffusion pore in the plastidic outer envelope may prove to be an over-simplification.

\section{Channels and porins in the outer membrane of Gram-negative bacteria}

It is generally assumed that the most important function of the outer membrane of Gram-negative bacteria is to form an additional and selective permeation barrier. Apart from secretion of proteins, DNA or polysaccharides (for an overview of export channels see Nikaido, 2003), outer membrane porins and channels thus mediate uptake of solutes (e.g., small hydrophilic nutrients). In brief, these membrane proteins can be subdivided into four classes: (i) classical porins; (ii) slow porins; (iii) specific channels; and (iv) TonB-dependent receptors/gated channels (Klebba and Newton, 1998; Nikaido, 2003). All these channel pores are $\beta$-barrels, formed by amphipathic transmembrane $\beta$-strands, and are exclusively found in the outer membrane but not the cytoplasmic membrane (Koebnik et al., 2000). Due to the elucidation of crystal structures, electrophysiological characterisation in planar lipid bilayers and osmotic swelling assays of liposomes, molecular dynamics simulations, and knowledge of prokaryotic genome sequences, diffusion of solutes through these bacterial channels can be described on the molecular level. In the following we introduce these protein classes by describing prototype members from the model prokaryote Escherichia coli as reviewed in detail by Nikaido (2003).

\section{Classical porins}

In general, a porin is defined as a water-filled membrane pore that forms a non-specific diffusion channel for solutes of $\leq 600 \mathrm{Da}$. Diffusion rates are proportional to the concentration difference across the membrane. OmpF, OmpC, and PhoE, three classical porins of Escherichia coli, have been characterised in detail. The porin channel is formed by a protein monomer of $39-40 \mathrm{kDa}$, which builds a $\beta$-barrel that consists of 16 strongly tilted $\beta$-sheets. However, in a functional porin, three of these channels are clustered together by one of the loops connecting the $\beta$-strands (Figure 1A). Usually these loops are short turns at the periplasmic side of the porin, but longer at the side facing the extracellular medium. The longest, external stretch (loop 3) folds into the pore, thereby narrowing the channel and building the so-called 'eyelet' of the porin with a diameter of $0.7-1.1 \mathrm{~nm}$ as determined for OmpF. Amino acid residues lining the channel wall in this eyelet influence the diffusion characteristics. While OmpF and OmpC prefer cations, PhoE is anion-selective, although their primary sequence is $72 \%$ similar. Cationic and anionic amino-acid residues of the very stable constriction eyelet are placed in opposite sides of the channel and thus orient the water molecules inside the pore in a highly directional manner. Furthermore, the external face of the $\beta$-barrel is occupied by lipophilic side chains, and aromatic amino acids are found at the outer and inner interfaces of outer membrane proteins in general. Interestingly, the C-terminus of porins invariably ends with a phenylalanine (or sometimes tryptophan), which is important for correct insertion and assembly of the porins into the outer membrane (Struyve et al., 1991; De Cock et al., 1997). The single channel conductance of a functional OmpF trimer is between 2 and $3 \mathrm{nS}$ and that of a monomer is $0.7 \mathrm{nS}$ (in $1 \mathrm{M} \mathrm{KCl}$ ), while the pore diameter of a monomer is estimated to be $1.2 \mathrm{~nm}$ [from sugar diffusion and polymer (polyethylene glycol, PEG) exclusion assays]. OmpC is slightly smaller but otherwise almost identical to OmpF. NmpC, OmpN, and OmpD are other classical porins of Enterobacteriaceae (Nikaido, 2003, and references therein). OmpG (34.9 kDa) of $E$. coli, however, is an unusual classical porin, since it does not form oligomers and lacks loop 3, and thus produces quite a large channel. It is most likely that OmpG thus functions in the uptake of larger oligosaccharides.

Because the E. coli porins described are very similar, gene expression and post-translational modifications are regulated very tightly to provide specific function and control diffusion of solutes (for an overview see Nikaido, 2003). PhoE, for example, is expressed only under phosphate starvation, and environmental factors such as $\mathrm{pH}$, polyamines, carbon sources and anaerobiosis regulate $\mathrm{OmpF}$ and $\mathrm{OmpC}$ function. It is generally assumed that the porins rest in an open state at a membrane potential of approximately $0 \mathrm{mV}$. In vitro the channels can be closed at voltages greater than $100 \mathrm{mV}$, but since there is no large electrical potential across the outer membrane of bacteria, this mechanism is most likely negligible in vivo. Therefore, other mechanisms, e.g., transcriptional and post-translational regulation, are likely to maintain the permeability barrier of the outer membrane. In the 
A

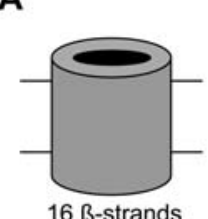

OmpF

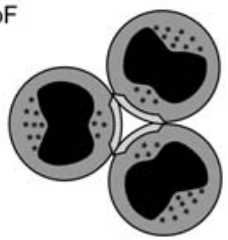

B

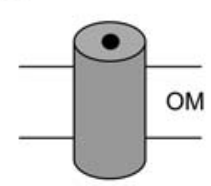

OmpA

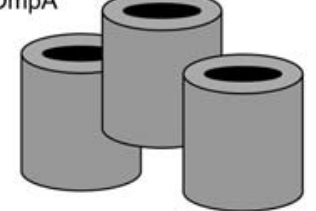

8 ß-strands 16 ß-strands

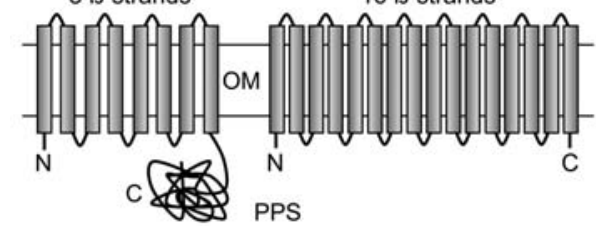

C

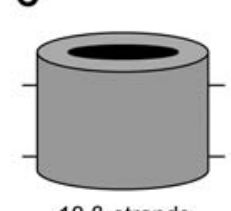

LamB

18 ß-strands

D

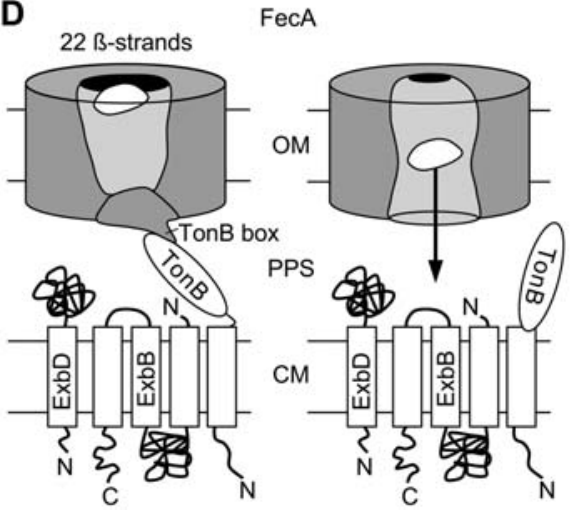

natural habitat of bacteria, the external loops interact with and are exposed to elements of the external world (e.g., chemicals, antibiotics, antibodies, phages). Therefore, these regions undergo very rapid mutagenesis. Thus, phylogeny based on the primary sequences of those porins alone is not feasible, and has to include structural and functional conservation.

\section{Slow porins}

Slow porins are very different from the classical porins, since diffusion of small solutes is much slower (by up to a factor of 50), but in contrast they allow larger solutes to pass through the channel. The major slow porin of $E$. coli is OmpA (37.2 kDa), while its homologue OprF from Pseudomonas aeruginosa was the first of this family identified and extensively discussed in the literature to understand how wider channels show slower diffusion of solutes (for an overview see Nikaido, 2003). This discrepancy is explained by the so-called 'two conformer' model, which is supported by most of the experimental
Figure 1 Membrane topology of porins and channels in the outer membrane of Gram-negative $E$. coli.

All models are simplified cartoons of the detailed structures depicted in the review by Nikaido (2003). (A) Left: the classical porin OmpF builds a $\beta$-barrel pore (black) in the outer membrane that consists of 16 antiparallel $\beta$-strands. Right: a functional channel is composed of three OmpF monomers as viewed from the top. This trimer is assembled by the interaction of loop regions connecting the $\beta$-strands (light grey). As indicated by asterisks, the extended loop 3 from the top folds into the pore, building the so-called 'eyelet' and narrowing the channel. (B) The current model for the function of the slow porin OmpA involves two conformations. Left: in the non-transporting but preferred conformation, the $\mathrm{N}$-terminal part of the protein forms $8 \beta$ sheets in the outer membrane (OM). The C-terminus extends into the periplasmic space (PPS) and interacts with the peptidogylcan layer. Right: a minority of proteins that tend to form loosely connected oligomers, however, can change into barrels of 16 continuous $\beta$-sheets and thereby open a pore. (C) Left: specific channels such as LamB are formed by $18 \beta$-strands. Middle: in a view from the top, it is evident that loop 3 (asterisks) folds into the channel and generates quite a narrow constriction zone compared to the porins in (A). Right: side view of a bisected LamB channel. Specificity for maltose is generated by the so-called 'greasy slide', which consists of six aromatic amino acids (black dots) lining the channel wall. During transport the sugar molecules interact with the aromatic side chains of these amino acids. (D) Simplified model of the action of FecA, a TonBdependent $\mathrm{Fe}^{3+}$-citrate receptor/gated channel. Left: in the nontransporting mode, a globular $\mathrm{N}$-terminal domain of the $22-\beta$-sheet FecA protein in the OM plugs the channel. When the ligand $\mathrm{Fe}^{3+}$-citrate (white) binds from the external side to the FecA receptor, a conformational change is induced that allows interaction of the TonB box at the N-terminus with the TonB protein in the PPS. For this interaction, TonB, which is anchored in the cytoplasmic membrane (CM), has to be energised via a proton gradient. In a hypothetical model this proton gradient is channelled by a complex of the membrane proteins ExbD, ExbB, and TonB. Right: in turn, interaction with TonB results in large conformational changes in FecA. First, the ligand-binding site is closed by external loops on the top of the protein, then removal of the plug gates the channel and allows transport of the ligand into the PPS.

evidence (Figure 1B). According to this model the majority of OmpA/OprF proteins are in the closed conformation, where the $\mathrm{N}$-terminal part of the protein is folded into eight $\beta$-sheets. The globular $\mathrm{C}$-terminus extends into the periplasm and interacts with the peptidoglycan layer, thereby stabilising the cell envelope, which is its primary function. However, a minority of the proteins can form a continuous $\beta$-barrel structure, with $16 \beta$-sheets needed to build an open, non-specific channel. Similar to the classical porins, this channel fraction tends to form oligomers. The fact that these open porins are underrepresented explains why diffusion of solutes is slow. In E. coli, which has the classical high-permeability porins, opened slow porins should have no functional consequence. In some pseudomonads lacking classical porins, however, this fraction is assumed to function as the major nonspecific porin. In addition to OmpW (22.9 kDa) from $E$. coli and homologues from other bacteria, several outer membrane proteins exist with only eight $\beta$-sheets. Although it is hypothesised that some of these proteins function as channels, it is still unclear how these small proteins could build sizeable pores. Thus, they rather might function as simple anchors or receptors, as 
reported for OmpW, which is described as a colicin receptor (Pilsl et al., 1999).

\section{Specific channels}

Specific channels catalyse the diffusion of selected nutrients, since they specifically bind transported solutes. Thus, diffusion along the concentration gradient across the membrane becomes specific and more efficient. LamB, the phage lambda receptor protein in $E$. coli, is the prototype for specific channels and catalyses the uptake of maltose, the most important carbon source for E. coli. Similar to the classical porins, LamB clusters into a homo-oligomeric trimer, but each of the monomeric channels of $49.9 \mathrm{kDa}$, which form a $\beta$-barrel with 18 $\beta$-strands, functions independently (Figure 1C). The external loop 3, together with loops 1 and 6, folds into the channel, thus generating a constriction zone of $0.5-0.6 \mathrm{~nm}$, which is much narrower than that of the porins (approx. 0.7-1.2 nm). In addition, the single-channel conductance of $0.16 \mathrm{nS}$ (in $1 \mathrm{M} \mathrm{KCl}$ ) is significantly lower than for porins. Specificity for maltose and higher oligosaccharides of the maltose series is mediated by the so-called 'greasy slide', consisting of six aromatic amino acids that line the entire channel wall (see Figure 1C). Transported sugar residues specifically interact with the aromatic rings of these amino acids. Although sucrose, for example, binds to the greasy slide, it is not transported since its fructose residue is prevented from entering the channel by the constriction region. However, under limiting carbon conditions, LamB is able to transport glucose, lactose, arabinose and glycerol.

For uptake of sucrose, E. coli uses the channel ScrY (55.4 kDa), which is homologous to LamB and transports a wide variety of sugars (e.g., glucose, fructose, arabinose, maltose, lactose, raffinose, maltodextrins). The 18-stranded $\beta$-barrel of ScrY is significantly wider $(0.85-1.1 \mathrm{~nm})$ than that of LamB, and the amino acids of the LamB constriction zone that inhibit fructose entry are absent in ScrY. Similar to LamB, the ScrY channel contains the greasy slide, but in contrast the protein possesses an $\mathrm{N}$-terminal extension, which is supposed to extend this greasy slide into the periplasm.

Nucleoside transport in E. coli is mediated by the specific channel Tsx (31.4 kDa), which builds a 14-strand $\beta$-barrel structure and does not form oligomers. Furthermore, the external loop 3 is short, and therefore loops 6 and 7 are relatively large and are important in substrate recognition. Another small channel protein that functions as a monomer is FadL ( $46 \mathrm{kDa}$ ), which mediates uptake of long-chain fatty acids via a 20 -strand $\beta$-barrel. Thus, Tsx and FadL are somewhat reminiscent to the classical but monomeric porin OmpG.

\section{TonB-dependent receptors/gated channels}

Insoluble ferric iron $\left(\mathrm{Fe}^{3+}\right)$ is transported into bacterial cells in the form of iron chelates (for overviews of bacterial iron transport systems in the outer membrane see Andrews et al., 2003; Ferguson and Deisenhofer, 2004; Cartron et al., 2006; Grass, 2006). Microbial iron chelators are called siderophores. However, $\mathrm{Fe}^{3+}$-siderophores, as well as vitamin $B_{12}$, are too large to pass through the classical porins or specific channels of the bacterial outer membrane. Therefore, prokaryotes utilise TonB-dependent receptors for uptake of these large molecules. E. coli has BtuB $(68.4 \mathrm{kDa})$ for vitamin $\mathrm{B}_{12}$ transport and six receptors for $\mathrm{Fe}^{3+}$-siderophore transport (e.g., FepA for $\mathrm{Fe}^{3+}$-enterobactin, FhuA for ferrichrome and FecA for $\mathrm{Fe}^{3+}$-citrate). These receptors bind the respective ligands with high affinity, which allows interaction of the receptor channel with the TonB protein in the periplasmic space (Figure 1D). The action of TonB, however, requires the cytoplasmic membrane to be energised via a proton gradient. This energy is then transferred to the outer membrane receptor with the assistance of two cytoplasmic membrane proteins (ExB and ExD), thereby gating the channel. The crystallographic structure of FecA, with and without bound $\mathrm{Fe}^{3+}$ citrate, revealed that the receptor channel is built of 22 $\beta$-strands. The $\mathrm{N}$-terminal domain of the $85.3-\mathrm{kDa}$ protein contains a TonB-binding box at its end and forms a globular structure that plugs the channel. The ligands bind to a site located on the external face of the protein, which contains portions of the plug and the $\beta$-barrel. Ligand binding results in a conformational change that allows interaction between the TonB box of FecA and TonB. In the current model (Figure 1D), transport is then initiated by closing of the receptor sites via movement of the external loops of the $\beta$-barrel. This is assumed to be followed by large conformational changes caused by the energised TonB, which in turn removes the plug and opens the channel pore.

\section{Porins in the outer membrane of cyanobacteria}

Since a cyanobacteria-like cell was the ancestor of today's plant chloroplasts (Moreira et al., 2000; Palmer, 2000), we include a short overview of porins in their Gram-negative type cell walls. Upon reconstitution into lipid bilayers, cell wall preparations and outer membrane protein extracts from the cyanobacteria Anabaena variabilis and Synechocystis PCC 6714 were shown to produce ion-permeable channels with single-channel conductance of 0.3 and $3.5 \mathrm{nS}$ (Anabaena) and 0.3 and 2.2 nS (Synechocystis), respectively (Benz and Böhme, 1985; Jürgens and Benz, 1989). In the strain Synechococcus PCC 6301, the outer membrane polypeptide pattern is dominated by a band of $52 \mathrm{kDa}$. The corresponding proteins, called SomA (Umeda et al., 1996) and SomB (Hoiczyk and Hansel, 2000), were identified to form trimeric homo-oligomers (140 kDa), which decompose into monomers of $52 \mathrm{kDa}$ upon heating in SDS (Hansel and Tadros, 1998). A single-channel conductance of $0.4 \mathrm{nS}$ for SomA and $0.9 \mathrm{nS}$ for a mixed preparation of SomA/SomB (in $1 \mathrm{M} \mathrm{KCl}$ ) was determined in lipid bilayers. In the genome of the model cyanobacterium Synechocystis PCC 6803, six SomA homologues have been identified (Hoiczyk and Hansel, 2000). Although they do not show sequence similarity to porins of other organisms, they might represent the low conductance channel described for cyanobacterial outer membranes. However, so far we can only speculate on the solutes they transport and whether this conductance 
Table 1 Permeability properties of OEP channels in the outer envelope of pea chloroplasts.

\begin{tabular}{lcccc}
\hline & OEP16 & OEP21 & OEP24 & OEP37 \\
\hline$\Lambda$ & $1.2 \mathrm{nS}$ & $0.95 \mathrm{nS}$ & $2.1 \mathrm{nS}$ & $0.5 \mathrm{nS}$ \\
& $(1 \mathrm{M} \mathrm{KCl})$ & $(1 \mathrm{M} \mathrm{NaCl})$ & $(1 \mathrm{M} \mathrm{KCl})$ & $(250 \mathrm{mM} \mathrm{KCl})$ \\
$\mathrm{P}_{\text {ion } 1} / \mathrm{P}_{\text {ion2 }}$ & $\mathrm{P}_{\mathrm{K}^{+}} / \mathrm{P}_{\mathrm{Cl}^{-}}$ & $\mathrm{P}_{\mathrm{Cl}^{-}} / \mathrm{P}_{\mathrm{K}^{+}}$ & $\mathrm{P}_{\mathrm{Ca}^{2}+} / \mathrm{P}^{+} / \mathrm{P}_{\mathrm{Cl}^{-}}$ & $\mathrm{P}_{\mathrm{K}^{+}} / \mathrm{P}_{\mathrm{Cl}^{-}}$ \\
& $6.5: 1$ & $5: 1$ & $3: 1: 0.27$ & $14: 1$ \\
$d(\mathrm{~nm})$ & $\sim 1.0$ & $2.36(\mathrm{v})$ & $2.5-3.0$ & $3.0(\mathrm{v})$ \\
& & $0.99(\mathrm{rz})$ & & $1.5(\mathrm{rz})$ \\
\hline
\end{tabular}

All properties depicted were determined for recombinant proteins reconstituted into lipid bilayers as described for OEP16 (Pohlmeyer et al., 1997), OEP21 (Bölter et al., 1999; Hemmler et al., 2006), OEP24 (Pohlmeyer et al., 1998), and OEP37 (Goetze et al., 2006). The single-channel conductance $(\Lambda)$ was measured in symmetrical solutions of the salt concentrations indicated. The relative permeability of the channels $\left(P_{\text {ion } 1} / P_{\text {ionn }}\right)$ is shown. Please note that OEP21 is anion-selective, while all other channels are cation-selective and OEP24 prefers divalent $\mathrm{Ca}^{2+}$ instead of monovalent $\mathrm{K}^{+}$ions. For OEP16, OEP21, and OEP24 the pore diameter (d) was estimated according to conductivity measurements and the permeation characteristics of solutes. For OEP37 the pore dimensions were determined using the polymer (PEG) exclusion method. Refined electrophysiological analyses revealed that the OEP21 and OEP37 channels most likely have an hourglass-shaped topology, with a wider vestibule (v) and narrowed restriction zone (rz).

is produced by trimeric or monomeric porins (Hoiczyk and Hansel, 2000; Nikaido, 2003). Besides the typical porin region with 14-16 membrane-spanning $\beta$-sheets, the SomA family of proteins contains an additional $\mathrm{N}$ terminally located domain, which most likely connects the proteins with the peptidoglycan layer or secondary cell-wall polymers. Thus, this topology is reminiscent of the slow porin OmpA of E. coli (see above). Apart from the previously mentioned Omp85-like $\beta$-barrels, so far these porins are the only channel-building proteins assigned to the outer membrane of cyanobacteria.

\section{OEPs: solute and metabolite channels in the outer envelope of plastids}

Solute transport across the outer envelope of chloroplasts is mediated by a set of channels that are unique to plastids and have distinct substrate specificities as determined by osmotic swelling of liposomes with reconstituted proteins. These channel-forming proteins are called OEPs. OEP16, OEP21, OEP24 and OEP37 classified according to their molecular weight - were all isolated as protein bands from fractionated outer envelope membranes of pea (Pisum sativum) chloroplasts. Thus, they represent abundant proteins in this membrane system. As usual for OEPs, none of them contains a classical chloroplast transit peptide. In addition to the preprotein-conducting channel Toc75 (Hinnah et al., 1997), these OEPs have been functionally characterised in vitro by electrophysiological measurements in artificial lipid bilayers (see Table 1 for details). They all represent highconductance solute channels with highest open probability at $0 \mathrm{mV}$ and thus resemble their ancestors in the outer membrane of Gram-negative bacteria, as described above. Their distinct substrate specificities indicate separate roles in different metabolic processes, challenging the notion that they are non-specific diffusion pores.

The dicotyledonous model plant Arabidopsis thaliana is currently used to study the in vivo function of the OEP proteins and to elucidate the evolution and regulation of the distinct OEP families. Whereas similar OEPs are present in the genomes of all land plants, including monocots such as rice and maize and the moss Physcomitrella patens, sequence homologues could not be identified in cyanobacteria, the progenitors of chloroplasts, or in other Gram-negative bacteria. Thus, as mentioned above, the high mutation rate in the amino acid sequence of bacterial porins and channels, in particular in domains facing the external medium, makes traditional phylogeny based solely on the primary sequence impossible. Therefore, assays combining structural and functional features have to be used to identify the ancestral OEP proteins. In the following, we review the four OEP protein families characterised so far and compare their properties to the porins and channels described for Gram-negative bacteria.

\section{OEP16, a channel specific for amino acids and amines}

The outer envelope protein OEP16 isolated from pea chloroplasts (Pohlmeyer et al., 1997) is integral to the membrane, as confirmed by its resistance to extraction at basic $\mathrm{pH}$ or under high salt conditions. The protein is present in different plastid types (etioplasts, proplastids, amyloplasts, and chloroplasts) of roots, shoots, and leaves of pea plants and was shown to form dimers in outer envelopes. Recombinant Ps-OEP16, reconstituted into lipid bilayers, forms a slightly cation-selective, highconductance channel. The conductance of $1.2 \mathrm{nS}$ determined in $1 \mathrm{M} \mathrm{KCl}$ (Table 1 ) is similar to that of porins $(0.7$ nS for OmpF monomers) from the outer membrane of Gram-negative bacteria. However, in contrast to these unspecific diffusion channels, Ps-OEP16 in liposome swelling assays displayed a strikingly high selectivity for amino acids and amines. The predicted OEP16 pore has a diameter of approximately $1.0 \mathrm{~nm}$, but is impermeable to 3-phosphoglycerate and uncharged sugars (fructose, glucose, sucrose, sorbitol). Thus, in this respect OEP16 rather resembles a specific channel such as the sucrosetransporting ScrY (pore diameter 0.85-1.1 nm). Furthermore, the OEP16 protein shows sequence and secondary structure similarity to components of the protein translocase of the inner mitochondrial membrane (Tim17, Tim22, and Tim23) and to a lesser extent to LivH, an amino acid transporter in the cytosolic membrane of E. coli (Rassow et al., 1999). Like these membrane transporters, OEP16 forms four $\alpha$-helical transmembrane domains according to circular dichroism studies (Figure 2A, Linke et al., 2004). This is rather unusual, since the channel proteins in the outer membranes of mitochondria (VDAC) and Gram-negative bacteria (see above) are exclusively built by $\beta$-barrels. In addition, all other plastid OEPs consist of membrane-spanning $\beta$-sheets (see below) inherited from the outer membrane of Gram-negative bacteria. In contrast, OEP16 seems to have evolved from the plasma membrane of either the bacterial endo- 
A
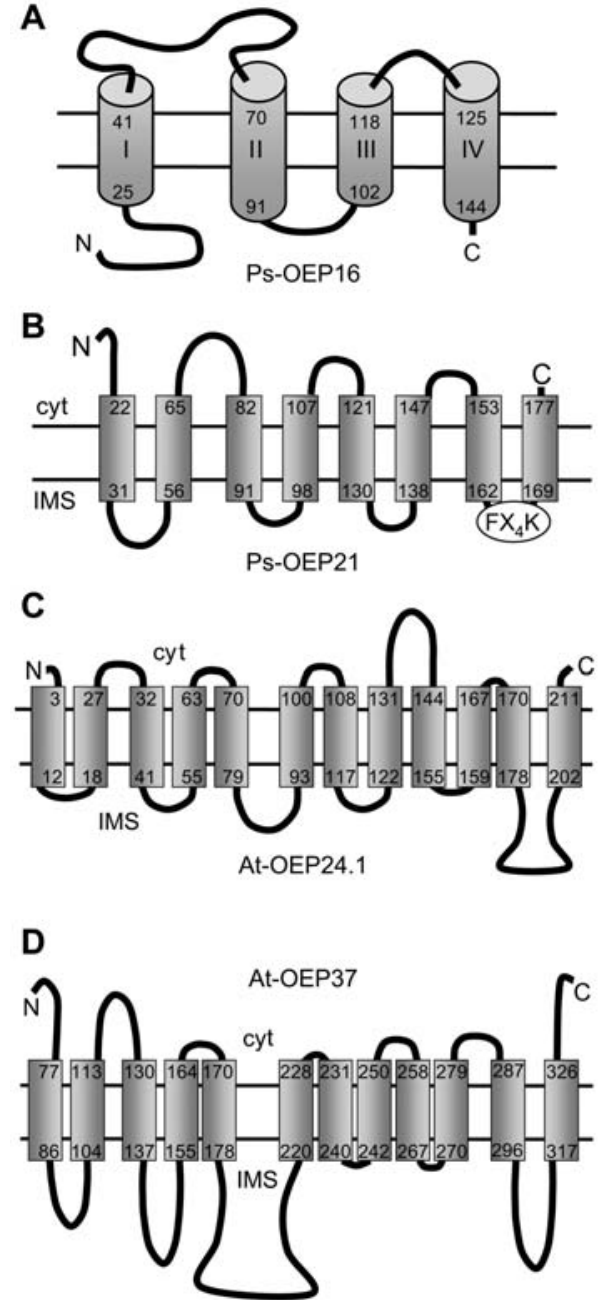

Figure 2 Membrane topology of OEP channels in the outer envelope of chloroplasts.

The polypeptide chains are depicted as solid lines, $\alpha$-helices as cylinders, and $\beta$-sheets as rectangles. The numbers of the first and last amino acids of each membrane-spanning domain are indicated. If known, the orientation of the proteins with respect to the cytosol (cyt) and intermembrane space (IMS) is indicated. (A) According to Linke et al. (2004), OEP16 from pea contains four $\alpha$-helical transmembrane domains. Helices II and IV are determined to be three to five amino acids longer than helices I and III. The loop connecting helices I and II, which most likely form the channel pore, is of unusual length and thus might form part of a selectivity filter or function in substrate recognition. (B) Ps-OEP21 consists of eight transmembrane $\beta$-sheets (Hemmler et al., 2006). The potential ATP-binding domain $\mathrm{FX}_{4} \mathrm{~K}$ is localised in the loop between $\beta$-sheets 7 and 8 and faces the intermembrane space. (C) Schleiff et al. (2003) modelled the $\beta$-sheet topology of the Arabidopsis protein At-OEP24.1. The membrane channel is most likely built from $12 \beta$-sheets. Two extended loops that connect $\beta$-sheets 8-9 and 11-12 face the cytosol and intermembrane space, respectively. (D) The longest loop of the $\beta$-barrel protein At-OEP37, which consists of $12 \beta$-strands (Schleiff et al., 2003), is located between $\beta$-sheets 5 and 6 and most likely extends into the intermembrane space.

symbiont or the eukaryotic host. Most likely, the functional OEP16 channel is formed by homo- or hetero-oligomers (see Arabidopsis isoforms below), in which helix 1 in combination with helix 2 of Ps-OEP16 builds the pore-forming region (Pohlmeyer et al., 1997; Steinkamp et al., 2000; Linke et al., 2004). As described for $E$. coli porins and the specific channel LamB, OEP16 possesses a stretched loop region connecting helices 1 and 2 (see Figure 2A) that might fold into the pore and thus provide a selectivity filter or mediate substrate recognition. However, to date it is not known whether this loop faces the cytosol or the intermembrane space. Interestingly, as reported for porins in the outer membrane of Gram-negative bacteria, protein sequences of the isoforms OEP16.1 and OEP16.2 in pea, Arabidopsis and rice (see below) end with the aromatic amino acids tyrosine or phenylalanine at their very C-terminus.

The model plant Arabidopsis has three OEP16 isoforms in chloroplasts, namely At-OEP16.1, At-OEP16.2, and At-OEP16.4 (Murcha et al., 2007; Philippar et al., 2007). The protein At-OEP16.3, originally designated as a member of the OEP16 proteins, is localised in mitochondria and it is now clear phylogenetically that it does not belong to the OEP16 subfamily. Instead, At-OEP16.3 belongs to the large but rather loose superfamily of plant PRAT (preprotein and amino acid transporters) proteins, which also includes the OEP16 and Tim17, 22, and 23 subfamilies (Drea et al., 2006; Murcha et al., 2007; Philippar et al., 2007). The isoform At-OEP16.1, most abundant in mature chloroplasts of light-grown leaves, shows the highest amino acid identity to OEP16 from pea and therefore most likely acts as an amino acid-selective channel in the outer envelope of Arabidopsis chloroplasts. Contradictory to a function in amino acid transport, some recent research proposes that OEP16.1 from barley and Arabidopsis acts as a precursor translocase for protochlorophyllide oxidoreductase (PorA) import into chloroplasts (Reinbothe et al., 2004, 2005; Pollmann et al., 2007). However, proper characterisation of single and double mutants of all Arabidopsis OEP16 isoforms does not support a function in PorA protein import (Philippar et al., 2007). Furthermore, expression of OEP16 is induced by light and cold, while PorA transcripts and proteins are completely absent upon illumination (Su et al., 2001; Philippar et al., 2007). Studies on cold induction of OEP16 in barley (Baldi et al., 1999) again suggest a function for OEP16.1 in amino acid transport, since cold acclimation, especially in cereals, requires an increase in free de novo synthesised amino acids and thus of channels that shuttle these compounds between the chloroplast and the cytosol. At-OEP16.2, in contrast, is very specifically expressed in non-photosynthetic plastids of late seed development and pollen (Drea et al., 2006; Philippar et al., 2007). During these stages of plant development, especially in storage cotyledons of the developing embryo, plastids primarily function in biosynthesis of amino acids and fatty acids needed for seed germination or pollen tube growth. Because expression of OEP16.2 seems to be under control of the phytohormone abscisic acid (Drea et al., 2006), which regulates seed dormancy, it is tempting to speculate that the role of OEP16.2 is in mediating transport of amino acids synthesised in the storage plastids of embryonic cotyledons. Transcripts of the isoform At-OEP16.4 are expressed ubiquitously, but at a low level (Philippar et al., 2007). Since OEP16 proteins tend to form dimers, OEP16.4 might be a partner for OEP16.1 and OEP16.2 in hetero-dimers of chloroplasts in leaves and plastids of seed cotyledons, respectively. 
In the physiological context, a function of OEP16 channels in the transport of amino acids thus seems more likely than translocation of the PorA precursor protein. However, detailed physiological studies of Arabidopsis OEP16 mutants in combination with characterisation in lipid bilayers of all three plastidic isoforms are necessary to unambiguously clarify this point.

\section{OEP21, a channel for phosphorylated carbohydrates, regulated from the intramembrane space}

The OEP21 protein from pea is present in different plastid types such as chloroplasts, etioplasts, and non-green plastids from roots. Ps-OEP21 has been shown to form a rectifying, anion-selective channel that is regulated by ATP and triosephosphates from the intermembrane space (Bölter et al., 1999). The channel has slightly lower conductivity $(0.95 \mathrm{nS}$ in $1 \mathrm{~m} \mathrm{NaCl})$ than OEP16 (see Table 1) and is permeable to inorganic phosphate and phosphorylated carbohydrates, central intermediates in the solute fluxes between chloroplasts and cytosol (e.g., triosephosphates, 3-phosphoglycerate, hexose phosphates). Very recently, refined electrophysiological analysis of the OEP21 channel properties revealed a $\beta$-barrel channel with a wider pore vestibule $(2.4 \mathrm{~nm})$ at the intermembrane site and a narrower filter pore of approximately $1 \mathrm{~nm}$ (Hemmler et al., 2006). In the open conformation, higher conductance at one site of the channel corresponds to current rectification in the direction from the intermembrane space to the cytosol. Thus, regarding selectivity, conductance and pore dimensions, OEP21 might be compared to the sucrose-transporting specific channel ScrY from E. coli (see above). Experiments on the protease resistance of OEP21 in the outer envelope in combination with computer-assisted modelling of the $\beta$-barrel topology confirmed previous circular dichroism data (Hemmler et al., 2006) revealing that the OEP21 monomer consists of eight $\beta$-strands with the $\mathrm{N}$ and $\mathrm{C}$-termini of the protein facing the cytosol (Figure $2 \mathrm{~B})$. Interestingly, only seven $\beta$-strands seem to contribute to the channel pore. A functional OEP21 channel therefore has to be composed of at least two monomeric units. However, it is not clear how these subunits could build one $\beta$-barrel. Thus, with respect to the topology, OEP21 is similar to the slow porin OmpA or to OmpW of E. coli (compare Figure 1B). Since the peptidoglycan layer in the periplasm of Gram-negative bacteria was lost during evolution (Hoiczyk and Hansel, 2000), OEP21 in chloroplasts in parallel might have lost the C-terminal domain of the OmpA-like proteins.

OEP21 from pea contains two binding sites for ATP, one high-affinity site at the centre of the pore, and a second, low-affinity site at the vestibule. The latter is represented by a C-terminal $\mathrm{FX}_{4} \mathrm{~K}$ motif in loop 7 connecting $\beta$-sheets 7 and 8 (Figure 2B), and faces the intermembrane space. Binding of ATP to this site blocks the channel, while binding to both sites decreases anion selectivity. Triose phosphates (TPS) can bind to both sites with the same affinity and thus compete with ATP. In consequence, increasing the TP:ATP ratio at the intermembrane space releases the current block and increases anion selectivity, resulting in a net efflux of TPs. Interest- ingly, one of the two Arabidopsis OEP21 proteins, At-OEP21.2, is equipped with the $\mathrm{FX}_{4} \mathrm{~K}$ motif, while the second isoform, At-OEP21.1, is lacking this regulatory site (Hemmler et al., 2006). Thus, future experiments with the two OEP21 isoforms in Arabidopsis should pinpoint the in planta regulation and function of OEP21.

\section{OEP24, a channel with specified function in a defined cell type}

The channel properties of the slightly cation-selective OEP24 from pea closely resemble those described for classical porins in Gram-negative bacteria (e.g., OmpF, see above). The 2.5-3.0-nm wide pore (Table 1) of the high-conductance channel $(2.1 \mathrm{nS}$ in $1 \mathrm{M} \mathrm{KCl})$ allows the passage of TPs, ATP, phosphate, dicarboxylate, and positively or negatively charged amino acids (Pohlmeyer et al., 1998). Furthermore, OEP24 can functionally replace the mitochondrial VDAC in VDAC-deficient yeast mutants (Röhl et al., 1999) and thus might represent a rather non-selective channel in the outer envelope. Secondary structure predictions and circular dichroism data suggest that OEP24 has a $\beta$-barrel-like structure containing 12 membrane-spanning $\beta$-strands (Figure 2C, Pohlmeyer et al., 1998; Schleiff et al., 2003). Two loops connecting the $\beta$-sheets of OEP2 4 extend somewhat into the cytosol and periplasmic space, respectively, and therefore might function in narrowing the channel like the external loops of bacterial porins (compare Figure 1A).

In Arabidopsis, two isoforms of OEP24 are present. Interestingly, mutation of At-OEP24.1 leads to a defect in pollen germination (Timper et al., unpublished data). In early pollen development, fatty acid biosynthesis in plastids supplies the growing pollen grain with the energy and lipid material needed for pollen tube emergence and growth (Yamamoto et al., 2003). Thus, the rather unspecific channel OEP24 seems to have a very specific and defined function that is triggered by expression in plastids during early pollen development. The isoform AtOEP24.2, in contrast, is expressed in late seed development. Again this is reminiscent of the transcriptional regulation of the classical porin proteins $\mathrm{OmpF}, \mathrm{OmpC}$ and PhoE in E. coli. In summary, the findings on Arabidopsis OEP24 prove the concept that metabolic fluxes across the outer envelope in vivo are mediated by OEP proteins, which are not redundant and unselective pores, but represent regulated channels essential for plastid function.

\section{OEP37, a new member of the chloroplast outer membrane channels}

Quite recently, OEP37 was described as a new member of the outer envelope channels (Goetze et al., 2006). Reconstituted recombinant OEP37 from pea forms a cation-selective, rectifying high-conductance channel (500 pS in symmetrical $250 \mathrm{~mm} \mathrm{KCl}$ ). The pore dimension could be estimated using the polymer (PEG) exclusion method (Table 1). Like OEP21, the channel pore reveals an hourglass-shaped form with different diameters for the vestibule $(3.0 \mathrm{~nm})$ and restriction zone $(1.5 \mathrm{~nm})$. OEP37 displayed affinity for the precursor of the chloroplast inner membrane protein Tic32 and for a synthetic pep- 
tide. Most likely, the channel pore of OEP37 is formed by $12 \beta$-strands (Figure 2D, Schleiff et al., 2003). In accordance with the porins and channels in the outer membrane of Gram-negative bacteria, OEP37 contains a long stretch (loop 5, see Figure 2D) connecting $\beta$-strands 5 and 6 that might be necessary for substrate specificity or recognition. However, as for its bacterial ancestors, this loop is not on the external face of the channel, but on the predicted side of the intermembrane space.

In Arabidopsis, transcripts of the single copy gene At-OEP37 are ubiquitously expressed throughout plant development and accumulate in early germinating seedlings, as well as during late embryogenesis. The plastid intrinsic protein OEP37 could be detected in isolated chloroplasts of cotyledons and rosette leaves. However, the knockout mutant oep37-1 shows that proper function is not essential for development of the mature plant (Goetze et al., 2006). In conclusion, OEP37 may constitute a novel peptide-sensitive channel in the outer envelope of plastids with function during embryogenesis and germination. Although the pore characteristics of OEP37 have been described in detail, substrate specificity and putative metabolite transport capacities await further characterisation. However, because of its expression in storage-type plastids of green cotyledons in seeds, it is tempting to speculate that OEP37 might transport stored fatty acid compounds and/or amino acids and act in concert with OEP16.2 or OEP24.2, which show similar expression patterns (see above).

In summary, the properties of the OEP channels described so far resemble several aspects of their bacterial ancestors. However, it is most likely that more channels and specifically regulated porins are required to shuttle all the metabolites and solutes necessary for the proper function of plastids across the outer envelope. Thus, these proteins still await discovery and description. Furthermore, in the near future it should be possible to circumstantiate the in planta function of the OEPs using the model plant Arabidopsis thaliana. Evaluation of crystal structures in combination with electrophysiological characterisation of all family members will help to delineate solute transport in molecular detail, as has been described for several porins in Gram-negative bacteria. From sequence comparison it is evident that outer membrane proteins of recent Gram-negative bacteria and in particular cyanobacteria show no direct homology to the chloroplast OEP channels. Thus, these organisms and plant organelles have diverged from their last common ancestor during evolution. However, the outer membrane channels and porins in today's cyanobacteria are not described so far on a detailed molecular, functional or structural level, which complicates direct comparison with the chloroplast OEPs. Furthermore, characterisation of the corresponding proteins in the model moss Physcomitrella patens or the green alga Chlamydomonas reinhardtii might help to descend the evolutionary ladder from higher plants towards the postulated common ancestor of cyanobacteria and chloroplasts. Thus, it is necessary to combine 'traditional' sequence-based phylogeny with detailed analyses of molecular structure and in vivo function to track the ancestral portrait gallery of the OEP channels.

\section{References}

Abrecht, H., Goormaghtigh, E., Ruysschaert, J.M., and Homble, F. (2000a). Structure and orientation of two voltage-dependent anion-selective channel isoforms. An attenuated total reflection Fourier-transform infrared spectroscopy study. J. Biol. Chem. 275, 40992-40999.

Abrecht, H., Wattiez, R., Ruysschaert, J.M., and Homble, F. (2000b). Purification and characterization of two voltagedependent anion channel isoforms from plant seeds. Plant Physiol. 124, 1181-1190.

Aljamal, J.A., Genchi, G., De Pinto, V., Stefanizzi, L., De Santis, A., Benz, R., and Palmieri, F. (1993). Purification and characterization of porin from corn (Zea mays L.) mitochondria. Plant Physiol. 102, 615-621.

Andrews, S.C., Robinson, A.K., and Rodriguez-Quinones, F. (2003). Bacterial iron homeostasis. FEMS Microbiol. Rev. 27, 215-237.

Atteia, A., van Lis, R., van Hellemond, J.J., Tielens, A.G.M., Martin, W., and Henze, K. (2004). Identification of prokaryotic homologues indicates an endosymbiotic origin for the alternative oxidases of mitochondria (AOX) and chloroplasts (PTOX). Gene 330, 143-148.

Baldi, P., Grossi, M., Pecchioni, N., Vale, G., and Cattivelli, L. (1999). High expression level of a gene coding for a chloroplastic amino acid selective channel protein is correlated to cold acclimation in cereals. Plant Mol. Biol. 41, 233-243.

Benz, R. (1994). Permeation of hydrophilic solutes through mitochondrial outer membranes: review on mitochondrial porins. Biochim. Biophys. Acta 1197, 167-196.

Benz, R. and Böhme, H. (1985). Pore formation by an outer membrane protein of the cyanobacterium Anabaena variabilis. Biochim. Biophys. Acta 812, 286-292.

Blumenthal, A., Kahn, K., Beja, O., Galun, E., Colombini, M., and Breiman, A. (1993). Purification and characterization of the voltage-dependent anion-selective channel protein from wheat mitochondrial membranes. Plant Physiol. 101, 579587.

Bölter, B. and Soll, J. (2001). Ion channels in the outer membranes of chloroplasts and mitochondria: open doors or regulated gates? EMBO J. 20, 935-940.

Bölter, B., Soll, J., Schulz, A., Hinnah, S., and Wagner, R. (1998). Origin of a chloroplast protein importer. Proc. Natl. Acad. Sci. USA 95, 15831-15836.

Bölter, B., Soll, J., Hill, K., Hemmler, R., and Wagner, R. (1999). A rectifying ATP-regulated solute channel in the chloroplastic outer envelope from pea. EMBO J. 18, 5505-5516.

Bos, M.P. and Tommassen, J. (2004). Biogenesis of the Gramnegative bacterial outer membrane. Curr. Opin. Microbiol. 7, 610-616.

Bowsher, C.G. and Tobin, A.K. (2001). Compartmentation of metabolism within mitochondria and plastids. J. Exp. Bot. 52, 513-527.

Bredemeier, R., Schlegel, T., Ertel, F., Vojta, A., Borissenko, L., Bohnsack, M.T., Groll, M., von Haeseler, A., and Schleiff, E. (2007). Functional and phylogenetic properties of the poreforming beta-barrel transporters of the Omp85 family. J. Biol. Chem. 282, 1882-1890.

Cartron, M.L., Maddocks, S., Gillingham, P., Jeremy, C.C., and Andrews, S.C. (2006). Feo-transport of ferrous iron into bacteria. BioMetals 19, 143-157.

Clausen, C., Ilkavets, I., Thomson, R., Philippar, K., Vojta, A., Möhlmann, T., Neuhaus, E., Fulgosi, H., and Soll, J. (2004). Intracellular localization of VDAC proteins in plants. Planta 220, 30-37.

Colombini, M., Yeung, C.L., Tung, J., and Konig, T. (1987). The mitochondrial outer membrane channel, VDAC, is regulated by a synthetic polyanion. Biochim. Biophys. Acta 905, 279-286. 
De Cock, H., Struyve, M., Kleerebezem, M., van der Krift, T., and Tommassen, J. (1997). Role of the carboxy-terminal phenylalanine in the biogenesis of outer membrane protein PhoE of Escherichia coli K-12. J. Mol. Biol. 269, 473-478.

Douce, R. and Joyard, J. (1990). Biochemistry and function of the plastid envelope. Annu. Rev. Cell Biol. 6, 173-216.

Drea, S.C., Lao, N.T., Wolfe, K.H., and Kavanagh, T.A. (2006). Gene duplication, exon gain and neofunctionalization of OEP16-related genes in land plants. Plant J. Cell Mol. Biol. 46, 723-735.

Ferguson, A.D. and Deisenhofer, J. (2004). Metal import through microbial membranes. Cell 116, 15-24.

Fischer, K., Weber, A., Brink, S., Arbinger, B., Schünemann, D., Borchert, S., Heldt, H.W., Popp, B., Benz, R., Link, T.A., et al. (1994). Porins from plants. Molecular cloning and functional characterization of two new members of the porin family. J. Biol. Chem. 269, 25754-25760.

Flügge, U.I. (2000). Transport in and out of plastids: does the outer envelope membrane control the flow? Trends Plant Sci. $5,135-137$.

Flügge, U.I. and Benz, R. (1984). Pore-forming activity in the outer membrane of the chloroplast envelope. FEBS Lett. 169, 85-89.

Gentle, I., Gabriel, K., Beech, P., Waller, R., and Lithgow, T. (2004). The Omp85 family of proteins is essential for outer membrane biogenesis in mitochondria and bacteria. J. Cell Biol. 164, 19-24.

Gentle, I.E., Burri, L., and Lithgow, T. (2005). Molecular architecture and function of the Omp85 family of proteins. Mol. Microbiol. 58, 1216-1225.

Godbole, A., Varghese, J., Sarin, A., and Mathew, M.K. (2003). VDAC is a conserved element of death pathways in plant and animal systems. Biochim. Biophys. Acta 1642, 87-96.

Goetze, T.A., Philippar, K., Ilkavets, I., Soll, J., and Wagner, R. (2006). OEP37 is a new member of the chloroplast outer membrane ion channels. J. Biol. Chem. 281, 17989-17998.

Grass, G. (2006). Iron transport in Escherichia coli: all has not been said and done. BioMetals 19, 159-172.

Hansel, A. and Tadros, M.N. (1998). Characterization of two pore-forming proteins isolated from the outer membrane of Synechococcus PCC 6301. Curr. Microbiol. 36, 321-326.

Heldt, H.W. and Sauer, F. (1971). The inner membrane of the chloroplast envelope as the site of specific metabolite transport. Biochim. Biophys. Acta 234, 83-91.

Hemmler, R., Becker, T., Schleiff, E., Bölter, B., Stahl, T., Soll, J., Goetze, T.A., Braams, S., and Wagner, R. (2006). Molecular properties of Oep21, an ATP-regulated anion-selective solute channel from the outer chloroplast membrane. J. Biol. Chem. 281, 12020-12029.

Hinnah, S.C., Hill, K., Wagner, R., Schlicher, T., and Soll, J. (1997). Reconstitution of a chloroplast protein import channel. EMBO J. 16, 7351-7360.

Hoiczyk, E. and Hansel, A. (2000). Cyanobacterial cell walls: news from an unusual prokaryotic envelope. J. Bacteriol. 182, 1191-1199.

Holden, M.J. and Colombini, M. (1993). The outer mitochondrial membrane channel, VDAC, is modulated by a protein localized in the intermembrane space. Biochim. Biophys. Acta 1144, 396-402.

Joyard, J., Teyssier, E., Miege, C., Berny-Seigneurin, D., Marechal, E., Block, M.A., Dorne, A.J., Rolland, N., Ajlani, G., and Douce, R. (1998). The biochemical machinery of plastid envelope membranes. Plant Physiol. 118, 715-723.

Jürgens, U.J. and Benz, R. (1989). Pore-forming activity of outer membrane extracts from the unicellular cyanobacterium Synechocystis PCC 6714. Z. Naturforsch. 44c, 147-151.

Klebba, P.E. and Newton, S.M. (1998). Mechanisms of solute transport through outer membrane porins: burning down the house. Curr. Opinion Microbiol. 1, 238-247.

Koebnik, R., Locher, K.P., and Van Gelder, P. (2000). Structure and function of bacterial outer membrane proteins: barrels in a nutshell. Mol. Microbiol. 37, 239-253.
Leister, D. (2003). Chloroplast research in the genomic age. Trends Genet. 19, 47-56.

Leister, D. (2005). Origin, evolution and genetic effects of nuclear insertions of organelle DNA. Trends Genet. 21, 655-663.

Linke, D., Frank, J., Pope, M.S., Soll, J., Ilkavets, I., Fromme, P., Burstein, E.A., Reshetnyak, Y.K., and Emelyanenko, V.I. (2004). Folding kinetics and structure of OEP16. Biophys. J. 86, 1479-1487.

Lopez-Juez, E. (2007). Plastid biogenesis, between light and shadows. J. Exp. Bot. 58, 11-26.

Lopez-Juez, E. and Pyke, K.A. (2005). Plastids unleashed: their development and their integration in plant development. J. Dev. Biol. 49, 557-577.

Mannella, C.A. (1998). Conformational changes in the mitochondrial channel protein, VDAC, and their functional implications. J. Struct. Biol. 121, 207-218.

Martin, W., Rujan, T., Richly, E., Hansen, A., Cornelsen, S., Lins, T., Leister, D., Stoebe, B., Hasegawa, M., and Penny, D. (2002). Evolutionary analysis of Arabidopsis, cyanobacterial, and chloroplast genomes reveals plastid phylogeny and thousands of cyanobacterial genes in the nucleus. Proc. Natl. Acad. Sci. USA 99, 12246-12251.

Moreira, D., Le Guyader, H., and Philippe, H. (2000). The origin of red algae and the evolution of chloroplasts. Nature 405, 69-72.

Murcha, M.W., Elhafez, D., Lister, R., Tonti-Filippini, J., Baumgartner, M., Philippar, K., Carrie, C., Mokranjac, D., Soll, J., and Whelan, J. (2007). Characterisation of the preprotein and amino acids transporter gene family in Arabidopsis. Plant Physiol. 134, 199-212.

Nikaido, H. (2003). Molecular basis of bacterial outer membrane permeability revisited. Microbiol. Mol. Biol. Rev. 67, 593-656.

Palmer, J.D. (2000). A single birth of all plastids? Nature 405, 32-33.

Pepper, A.E. (1998). Molecular evolution: old branches on the phytochrome family tree. Curr. Biol. 8, R117-R120.

Philippar, K. and Soll, J. (2007). Intracellular transport: solute transport in chloroplasts, mitochondria, peroxisomes and vacuoles, and between organelles. In: Plant Solute Transport, A.R. Yeo and T.J. Flowers, eds. (Oxford, UK: Blackwell Publishing), pp. 133-192.

Philippar, K., Geis, T., Ilkavets, I., Oster, U., Schwenkert, S., Meurer, J., and Soll, J. (2007). Chloroplast biogenesis: the use of mutants to study the etioplast-chloroplast transition. Proc. Natl. Acad. Sci. USA 104, 678-683.

Pilsl, H., Smajs, D., and Braun, V. (1999). Characterization of colicin S4 and its receptor, OmpW, a minor protein of the Escherichia coli outer membrane. J. Bacteriol. 181, 35783581.

Pohlmeyer, K., Soll, J., Grimm, R., Hill, K., and Wagner, R. (1998). A high-conductance solute channel in the chloroplastic outer envelope from pea. Plant Cell 10, 1207-1216.

Pohlmeyer, K., Soll, J., Steinkamp, T., Hinnah, S., and Wagner, R. (1997). Isolation and characterization of an amino acidselective channel protein present in the chloroplastic outer envelope membrane. Proc. Natl. Acad. Sci. USA 94, 95049509.

Pollmann, S., Springer, A., Buhr, F., Lahroussi, A., Samol, I., Bonneville, J.M., Tichtinsky, G., von Wettstein, D., Reinbothe, C., and Reinbothe, S. (2007). A plant porphyria related to defects in plastid import of protochlorophyllide oxidoreductase A. Proc. Natl. Acad. Sci. USA 104, 2019-2023.

Pottosin, I.I. (1992). Single channel recording in the chloroplast envelope. FEBS Lett. 308, 87-90.

Pottosin, I.I. (1993). One of the chloroplast envelope ion channels is probably related to the mitochondrial VDAC. FEBS Lett. 330, 211-214.

Rassow, J., Dekker, P.J., van Wilpe, S., Meijer, M., and Soll, J. (1999). The preprotein translocase of the mitochondrial inner membrane: function and evolution. J. Mol. Biol. 286, 105120. 
Raven, J. and Allen, J. (2003). Genomics and chloroplast evolution: what did cyanobacteria do for plants? Genome Biol. 4, 209.

Reinbothe, S., Quigley, F., Springer, A., Schemenewitz, A., and Reinbothe, C. (2004). The outer plastid envelope protein Oep16: role as precursor translocase in import of protochlorophyllide oxidoreductase A. Proc. Natl. Acad. Sci. USA 101, 2203-2208.

Reinbothe, S., Pollmann, S., Springer, A., James, R.J., Tichtinsky, G., and Reinbothe, C. (2005). A role of Toc33 in the protochlorophyllide-dependent plastid import pathway of NADPH: protochlorophyllide oxidoreductase (POR) A. Plant J. 42, 1-12.

Reumann, S., Inoue, K., and Keegstra, K. (2005). Evolution of the general protein import pathway of plastids. Mol. Membr. Biol. 22, 73-86.

Röhl, T., Motzkus, M., and Soll, J. (1999). The outer envelope protein OEP24 from pea chloroplasts can functionally replace the mitochondrial VDAC in yeast. FEBS Lett. 460, 491-494.

Rostovtseva, T. and Colombini, M. (1997). VDAC channels mediate and gate the flow of ATP: implications for the regulation of mitochondrial function. Biophys. J. 72, 1954-1962.

Schleiff, E., Eichacker, L.A., Eckart, K., Becker, T., Mirus, O., Stahl, T., and Soll, J. (2003). Prediction of the plant betabarrel proteome: a case study of the chloroplast outer envelope. Protein Sci. 12, 748-759.

Schmid, A., Kromer, S., Heldt, H.W., and Benz, R. (1992). Identification of two general diffusion channels in the outer membrane of pea mitochondria. Biochim. Biophys. Acta 1112, 174-180.

Soll, J. and Schleiff, E. (2004). Protein import into chloroplasts. Nat. Rev. Mol. Cell Biol. 5, 198-208.

Soll, J., Bölter, B., Wagner, R., and Hinnah, S.C. (2000). ... response: The chloroplast outer envelope: a molecular sieve? Trends Plant Sci. 5, 137-138.

Steinkamp, T., Hill, K., Hinnah, S.C., Wagner, R., Rohl, T., Pohlmeyer, K., and Soll, J. (2000). Identification of the pore-forming region of the outer chloroplast envelope protein OEP16. J. Biol. Chem. 275, 11758-11764.

Struyve, M., Moons, M., and Tommassen, J. (1991). Carboxy- terminal phenylalanine is essential for the correct assembly of a bacterial outer membrane protein. J. Mol. Biol. 218, 141-148.

Su, Q., Frick, G., Armstrong, G., and Apel, K. (2001). POR C of Arabidopsis thaliana: a third light- and NADPH-dependent protochlorophyllide oxidoreductase that is differentially regulated by light. Plant Mol. Biol. 47, 805-813.

Tetlow, I.J., Rawsthorne, S., Raines, C., and Emes, M.J. (2005). Plastid metabolic pathways. In: Plastids, S.G. Möller, ed. (Oxford, UK: Blackwell Publishing), pp. 60-125.

Umeda, H., Aiba, H., and Mizuno, T. (1996). SomA, a novel gene that encodes a major outer-membrane protein of Synechococcus sp. PCC 7942. Microbiology 142, 2121-2128.

Van der Heiden, M.G., Chandel, N.S., Li, X.X., Schumacker, P.T., Colombini, M., and Thompson, C.B. (2000). Outer mitochondrial membrane permeability can regulate coupled respiration and cell survival. Proc. Natl. Acad. Sci. USA 97, 4666-4671.

Vothknecht, U.C. and Soll, J. (2005). Chloroplast membrane transport: interplay of prokaryotic and eukaryotic traits. Gene 354, 99-109.

Wandrey, M., Trevaskis, B., Brewin, N., and Udvardi, M.K. (2004). Molecular and cell biology of a family of voltage-dependent anion channel porins in Lotus japonicus. Plant Physiol. 134, 182-193.

Waters, M. and Pyke, K. (2005). Plastid development and differentiation. In: Plastids, S.G. Möller, ed. (Oxford, UK: Blackwell Publishing), pp. 30-59.

Weber, A.P. (2004). Solute transporters as connecting elements between cytosol and plastid stroma. Curr. Opin. Plant Biol. 7, 247-253.

Weber, A.P.M., Schwacke, R., and Flügge, U.I. (2005). Solute transporters of the plastid envelope membrane. Annu. Rev. Plant Biol. 56, 133-164.

Yamamoto, Y., Nishimura, M., Hara-Nishimura, I., and Noguchi, T. (2003). Behavior of vacuoles during microspore and pollen development in Arabidopsis thaliana. Plant Cell Physiol. 44, 1192-1201.

Zizi, M., Forte, M., Blachly-Dyson, E., and Colombini, M. (1994). $\mathrm{NADH}$ regulates the gating of VDAC, the mitochondrial outer membrane channel. J. Biol. Chem. 269, 1614-1616. 